\title{
REVIEW OF METHODS FOR DIABETIC RETINOPATHY DETECTION AND SEVERITY CLASSIFICATION
}

\author{
Madhura Jagannath Paranjpe ${ }^{1}$, M N Kakatkar ${ }^{2}$ \\ ${ }^{I}$ ME student, Department of Electronics and Telecommunication, Sinhgad College of Engineering, University of Pune, \\ Pune, India \\ ${ }^{2}$ Professor, Department of Electronics and Telecommunication, Sinhgad College of Engineering, University of Pune, \\ Pune, India
}

\begin{abstract}
Diabetic Retinopathy is a serious vascular disorder that might lead to complete blindness. Therefore, the early detection and the treatment are necessary to prevent major vision loss. Though the Manual screening methods are available, they are time consuming and inefficient on a large image database of patients. Moreover, it demands skilled professionals for the diagnosis. Automatic Diabetic Retinopathy diagnosis systems can replace manual methods as they can significantly reduce the manual labor involved in the screening process. Screening conducted over a larger population can become efficient if the system can separate normal and abnormal cases, instead of the manual examination of all images. Therefore, Automatic Retinopathy detection systems have attracted large popularity in the recent times. Automatic retinopathy detection systems employ image processing and computer vision techniques to detect different anomalies associated with retinopathy. This paper reviews various methods of diabetic retinopathy detection and classification into different stages based on severity levels and also, various image databases used for the research purpose are discussed.
\end{abstract}

Keywords - Automatic Diabetic Retinopathy detection, computer vision, Diabetic Retinopathy, image databases, image processing, manual screening

\section{INTRODUCTION}

The population of diabetic patients has been increasing against the total world population. Uncontrolled and prolonged diabetes can damage the microvasculature of the vital organs of the body such as eyes and kidneys. The damage caused to the tiny blood vessels in the retina of the human eye, is known as Diabetic Retinopathy. Due to elevated amounts of glucose circulating through the body, the walls of blood vessels become damaged and several anomalies such as Microaneurysms, hemorrhages, Hard Exudates, Cotton wool spots start developing at various phases of retinopathy. The patient affected by Diabetic Retinopathy may not experience visual impairment until the disease has progressed to a severe stage, when the treatment is less effective. Therefore the early detection and the regular follow-ups is necessary to treat diabetic retinopathy.

The earliest symptoms of Retinopathy are the Micro aneurysms, which occur due to dilatations of the blood capillaries and they appear as dark red spots on the retina. Hemorrhages occur when the microaneurysms burst. Bright-yellow colored Lesions such as hard exudates occur as a result of fluid leaking into the retinal surface from the capillaries or from Microaneurysms. Another bright white colored lesions, called the soft Exudates or cotton wool spots occur occlusions of the nerve fibre layer. Diabetic Retinopathy is a progressive disease. The first stage of retinopathy is known as Non-Proliferative Retinopathy, during which the retinal lesions appear and increase as the disease progresses. Initially, at least one microaneurysm is seen. With the progression of the disease, the blood vessels become blocked and are short of blood supply. In an attempt to create new paths for blood supply, abnormal and fragile new blood vessels are formed on the surface of retina in the stage of Proliferative Retinopathy that might leak blood into retina causing permanent blindness. The various lesions associated with diabetic retinopathy are as shown in the figure below.

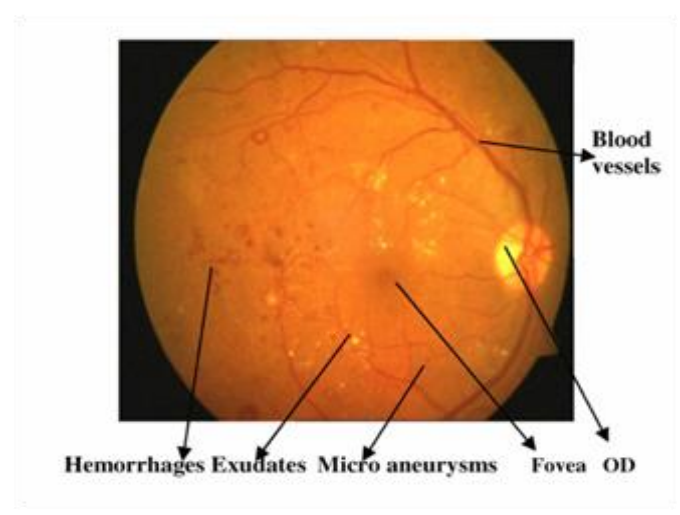

Fig. 1 Retinal lesions associated with diabetic retinopathy 
Fig.1 shows the retinal image of a diabetic eye with anatomical features such as the optic disc and blood vessels and Fovea, and the abnormal features such as hemorrhages, Exudates, Microaneurysms.

The process of Automatic Diabetic Retinopathy detection involves detection and segmentation of the abnormal features from the input images. The general block diagram for the automatic diabetic retinopathy detection and classification is as shown in Fig. 2. The input image from the retinal image database is preprocessed to extract the grayscale or green component of the image, noise removal and to enhance the contrast of the image for further processing. The next step is to localize the retinal components such as Optic Disc, Fovea and blood vessels. In the next step, abnormal features such as micro-aneurysms, hemorrhages and hard exudates and cottonwool spots are extracted. These features are analyzed with different techniques to perform severity classification of the disease as normal, mild, moderate, severe Non proliferative Retinopathy (NPDR) and Proliferative Retinopathy (PDR).

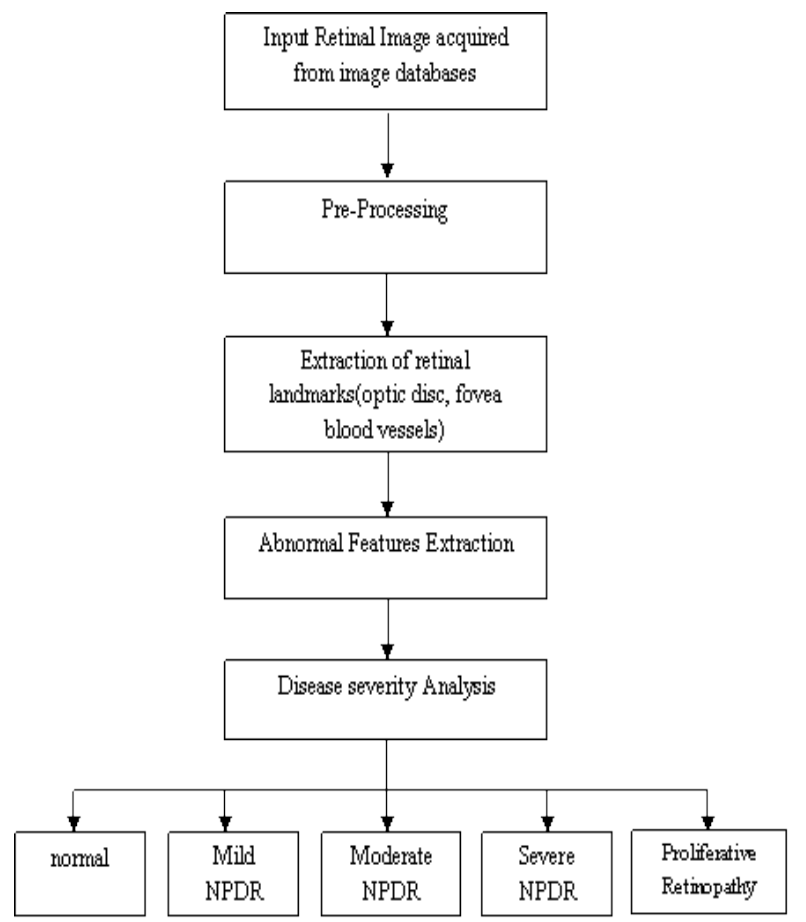

Fig.2 General Block diagram for diabetic retinopathy detection and severity classification

\section{LITERATURE REVIEW}

There are several approaches to diabetic retinopathy detection and classification. Many techniques based on mathematical morphology, neural networks, pattern recognition, region growing techniques, fuzzy C-means clustering, Gabor filter banks are available from the literature. Blood vessels were detected using two-dimensional matched filter [1]. Automatic extraction of the vasculature was done using a sparse tracking method [2]. Automatic detection and the classification of abnormalities on the vascular network was done by using Gabor filter bank outputs at several finer scales to obtain a scale and angle method of representation of energy variations, to classify the mild, moderate and severe stages of retinopathy [3]. Sinthanayothin developed a method based on recursive region growing methods and Moat operator to detect Hemorrhages, Microaneurysms and exudates [4]. Several Optic disc detection methods were proposed. Principal Component Analysis (PCA) based approaches were derived where the candidate regions for optic disc were derived by clustering of brighter pixels.PCA was applied to calculate the minimum distance between the image and its projection to find the optic disc center [5]. Optic Disc detection was also done using Hough Transform [6]. Microaneurysms and hemorrhages were detected using morphological operations with a structuring element and tophat transformation [7]. Mahalanobis classifier was used to identify hemorrhages and microaneurysms [8]. Image processing techniques in combination with pattern recognition techniques were used to detect microaneurysms and hemorrhages in fundus images [9]. Higher Gray level variations of the exudates and morphological reconstruction methods were used in the extraction of exudates [10]. Automatic exudates and cotton-wool spots detection system is developed in [11]. A neural network based approach was used exudates detection [12]. A fuzzy C-means clustering method [13] and computational intelligence based approach was proposed for detection of exudates [14]. Acharya [15] classified Diabetic Retinopathy stages as normal, mild, moderate, severe and proliferative. The feature extraction was done using Higher Order Spectra (HOS).A multi-layer perceptron was used to classify normal and diabetic retinopathy stages [4]. Kahai developed a decision support system, using bayes optimality criterion to detect Microaneurysms that enables the early detection of diabetic retinopathy [16]. Area and perimeter calculated from the RGB components of the blood vessels were used as features to classify normal, mild, moderate, severe and proliferative stages of retinopathy using a feed forward neural network [17]. Nayak et al. used features such as area of exudates and the area of blood vessels together with texture parameters and features are input to the neural network to classify images into normal, Non-Proliferative Retinopathy and Proliferative Retinopathy [18]. Automatic classification into normal, mild, moderate, severe and proliferative classes of Diabetic Retinopathy was done by calculating the areas of several features such as, hemorrhages, microaneurysms, exudates and blood vessel with support vector machine as classifier [19]. Automated diagnosis system is developed to detect retinal blood vessels, and pathologies, such as exudates and microaneurysms together with certain texture properties using image processing techniques. The area of lesions and texture features are then used to construct a feature vector that is input to the multiclass support vector machine (SVM) for classifying images into normal, mild, moderate, severe and proliferative categories [20]. 


\section{OVERVIEW OF DIABETIC RETINOPATHY DETECTION AND SEVERITY CLASSIFICATION}

Automated diabetic retinopathy detection is a computer vision problem. It involves the extraction of retinal lesions such as microaneurysms, hemorrhages, exudates. The accurate detection of these features is faced with some challenges.

1. Similarity of retinal lesions to the landmark features such as blood vessels, optic disc and the macula (fovea).

2. Varying illumination and contrast changes across the image.

The hemorrhages and microaneurysms to have same color as the blood vessels. Larger hemorrhages appear to have same color as that of blood vessel network, but have different geometrical features. But the smaller microaneurysms and hemorrhages might present similar color, contrast and geometrical features as the thin and smaller blood vessels, making it difficult to discriminate between them. The optic disc is a bright region with circular geometry, which shares the same color feature as that of the exudates. The fovea is a dark red region at the center of macula which is similar in color as that of micro-aneurysms and hemorrhages. These retinal components must be detected and localized and segmented such that anomalous features can be detected accurately.

Non uniform illumination and contrast variations across the image pose difficulties, as retinal feature extraction algorithms work accurately on images with good contrast and no background variations. Therefore, robust preprocessing operations are required to tackle this problem. The presence of non retinopathic features which may contain similar color, contrast as that of other retinal features, might lead to a non retinopathic feature be wrongly classified as a retinopathic feature. Presence of artifacts that might occur due to improper image acquisition conditions can be confused for a retinopathic feature. In some images, hemorrhages and exudates may exhibit very little color differences, which demands robust color normalization techniques.

The important steps in the detection and severity classification process are as follows:

1. Pre-processing for contrast enhancement and removal of noise.

2. Detection, Localization of the Optic Disc and its segmentation

3. Retinal vascular tree segmentation

4. Localization of fovea region

5. Abnormal Feature Extraction

6. Classification of different stages of Diabetic Retinopathy

7. Evaluation of the performance of classifier.

\subsection{Preprocessing}

The main aim of preprocessing methods is to achieve image normalization by attenuation of intensity variations in the input images. The original images contain non-uniform spatial variations across the image. Several models have been developed to attenuate such variations. Adaptive contrast enhancement techniques, mathematical model representation of the non uniformness of the image, image filtering techniques have been developed. Correction methods for non-uniform illumination, subtract the non-uniform component of the image from the original image to filter the variations. Several approaches assume the image to be consisting of foreground and background components. Retinal vasculature, lesions and the optic disc constitute the foreground and the remaining features constitute the background. Shade correction algorithms generate background approximation using mean filtering. Then the original green channel image is subtracted or divided by the background to achieve shade correction.

Since color is an important feature to differentiate between different lesions, normalization of color descriptor is important to minimize the color variations in the images. The alternative approach to reducing variations is the histogram equalization by redistribution of the gray levels to achieve uniform distribution of pixel intensities. Contrast enhancement methods enhance the contrast of the image and are usually applied on the low contrast images.

\subsection{Localization of the Optic Disc and Segmentation of the Disc}

To differentiate the exudates from the optic disc, localizing the disc and segmentation of the optic disc is essential. This process consists of finding approximate optic disc center. The problem is the distraction caused by other larger lesions such as exudates. In [1], the optic disc was considered to be a region having large cluster of bright pixels. Circular Hough transform and Principal Component Analysis are the other methods to detect the optic disc. Morphological operators followed by active contour model to segment the optic disc have also been used for optic disc segmentation.

\subsection{Detection of Fovea}

Fovea is the darkest region in the retina and its color is similar to blood vessels and microaneurysms and hemorrhages. Therefore the fovea must be localized. In [5], model based methods have been used.

\subsection{Segmentation of Retinal Blood Vessels}

Retinal vasculature segmentation is done by applying morphological operators and edge detection. The matched filter approach [1] is an improvement over the sobel edge operators and morphological operators. Vessel segmentation using mathematical morphology and curvature evaluation methods provided better results than matched filters. Supervised classification approaches using neural networks are based on pixel classification, in which a pixel feature vector is 
constructed for each pixel to classify the pixel to be belonging to vessel or non-vessel [4]. Techniques using PCA, Gabor filters and Bayesian classifiers are used in supervised classification.

\subsection{Feature Extraction for Diabetic Retinopathy}

After the optic disc, fovea and blood vessel network localization the exudates, hemorrhages and microaneurysms are extracted from the images.

1. Microaneurysms and hemorrhages detection: The detection of these dark red regions requires the removal of other brighter regions such as exudates and optic disc. Initially, Morphological operations using a structuring element, top-hat transformation were the methods used. Recursive region growing methods with the moat operator provided a sensitivity of $77.5 \%$ and specificity of $88.7 \%$. Pattern recognition techniques that compare the human and machine performance in detecting the abnormalities have been developed.

2. Detection of hard Exudates and cotton wool spots: Exudates that are formed due to lipid, protein accumulated over the retina and are brightly colored. Therefore, the other brighter regions i.e., blood vessel network and the optic disc must be removed before extracting exudates Recursive Region growing algorithms, which assume pixel adjacency in terms of similarity in gray levels, were used to detect the boundary of a region [4]. Other methods using morphological reconstruction, neural networks, Fuzzy c-means clustering and computational intelligence techniques are also used. Cotton wool spots are also the bright white colored features and a thresholding scheme was employed in [11] to differentiate between the two.

3. Texture information: Texture provides a measure of properties of an image, as smooth, coarse or regular with the uniform variation of pixel intensities. Structural, statistical and spectral are the three ways to measure the texture. Statistical methods employ the spatial relationship between the pixels intensities. Measures such as entropy, contrast, homogeneity, correlation, energy are extracted from the Gray level Cooccurrence matrix. Different texture measures that explain the gray-level variations existing among the features can be used to detect of Diabetic Retinopathy severity.

\subsection{Classification of Diabetic Retinopathy Severity}

In the Mild NPDR stage, at least one microaneurysm with or without hemorrhages, exudates might be present. The moderate NPDR consists of large numbers of hemorrhages and microaneurysms with the presence of exudates. In severe retinopathy, microaneurysms and hemorrhages occupying all four quadrants of the retina with vascular abnormalities In the most severe stage known as Proliferative Retinopathy, abnormal, new blood vessels grow on retinal surface. The different stages of retinopathy are as shown in the diagram below.
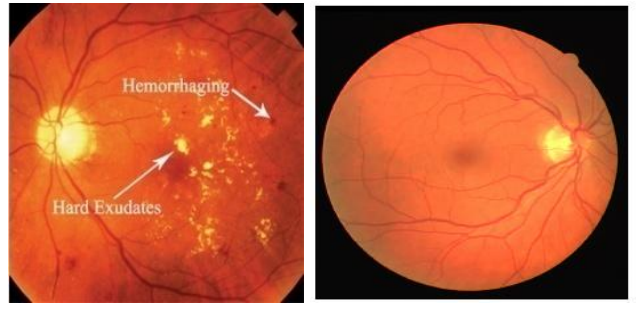

Fig 3(a) normal

Fig.3 (b) mild NPDR
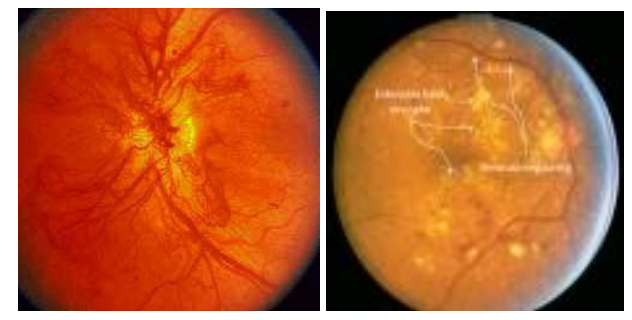

Fig.3 (c) moderate NPDR

Fig 3 (d) severe NPDR

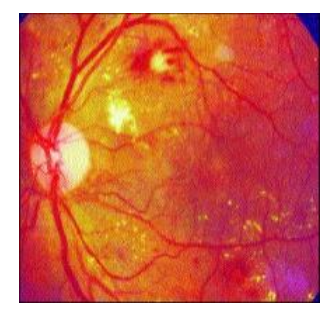

Fig.3 (e) PDR

After the features are extracted, according to the extent of features extracted, classification is done using different types of classifiers, such as neural networks, support vector machines. Efficiency of the classifier is calculated in terms of its efficiency to classify normal images into normal and abnormal images as abnormal. Area and perimeter of blood vessels were used as the input parameters to multilayer feed forward neural network for classification into different retinopathy stages. From the literature, area of blood vessels, exudates and texture properties are used to classify the stages as normal, nonProliferative Retinopathy (NPDR) and Proliferative Retinopathy (PDR). As seen from the literature, classification accuracy improves when all the features, i.e. blood vessels, microaneurysms, hemorrhages, exudates along with texture information are used in classification.

\subsection{Evaluation of the Performance of Classifier}

Several parameters such as True Positive (TP), True Negative (TN), False Positive (FP) and False Negative (FN) are calculated. These parameters are calculated by comparing the classifier outcome with the number of normal and abnormal images from the database. For an abnormal image, the result is true positive if the outcome of classification is abnormal and the result is False Negative (FN) if the classifier output is normal. 
For normal image, the result is True Negative (TN), if the classifier output is normal and False Positive (FP) if the classification outcome is abnormal. In a given image dataset, these parameters, TP,TN, FP, FN are used in the calculation of the accuracy, Sensitivity (SN) and specificity (SP). Performance of the classifier can be measured in terms of sensitivity, specificity and accuracy.

1. Sensitivity $=\mathrm{TP} /(\mathrm{TP}+\mathrm{FN})$

Sensitivity is measure of the percentage of abnormal images classified as abnormal.

2. Specificity $=\mathrm{TN} /(\mathrm{TN}+\mathrm{FP})$

Specificity gives the measure of normal images that are classified correctly as normal.

3. Accuracy $=(\mathrm{TP}+\mathrm{TN}) /(\mathrm{TP}+\mathrm{FN}+\mathrm{TN}+\mathrm{FP})$

It is the measure of total number of well classified normal and abnormal images.

\section{IMAGE DATASETS USED FOR THE EXPERIMENTATION AND RESEARCH}

The Efficiency and the performance evaluation of the algorithm used for the feature extraction can be analyzed if the results are compared with the Ground truth or manually labeled images available from the databases. The manual segmentations are created by handmade markings by trained specialists and ophthalmologists. The difference between the manual segmentations and the segmentation result of the algorithm evaluates the performance of the algorithm. There are several databases which contain ground truth results for blood vessel segmentations, Microaneurysm, exudates segmentations and to evaluate the efficiency of classification of diabetic retinopathy severity. The DRIVE database, (Digital Retinal Images for Vessel Extraction) is a publicly available database, and consists of 40 color fundus images, stored in the TIFF format. The database is divided into training and test images. Another database called the STARE also consists of manual vessel segmentations, for each of the images. DIARETDB1 and DIARETDB0 contain normal images and abnormal images containing symptoms of retinopathy. The images are $1500 \times 1152$ pixels wide and are stored in PNG format. Other important datasets are MESSIDOR, REVIEW and HEI-MED.

\section{CONCLUSIONS}

The generalized framework presented in this paper reviews the various methods and techniques used in the diabetic retinopathy detection and severity classification. Section II reviews various approaches and the methodologies taken up by the researchers to detect diabetic retinopathy. Section III presents the process of diabetic retinopathy detection and highlights different challenges associated with it. The section IV provides an overview of different datasets used for experimentation and research purpose.

\section{ACKNOWLEDGMENTS}

I wish to express my sincere thanks and gratitude to respected guide Mr. M. N. Kakatkar, Professor in Department of Electronics and Telecommunication Engineering, Sinhgad college of Engineering, Wadgaon (BK), Pune-41, for the motivation, technical suggestions and constructive criticism, which enabled to work harder towards excellence.

\section{REFERENCES}

[1]. Chaudhuri, S., Chatterjee, S., Katz, N., Nelson, M., and Goldbaum, M., Detection of blood vessels in retinal images using two-dimensional matched filters. IEEE Trans. Med. Imag.8 (3):263-269, 1989.

[2]. Grisan, I. E., Pesce, A., Giani, A., Foracchia, M., and Ruggeri, A., A new tracking system for the robust extraction of retinal vessel structure, 26th Annual International Conference of the IEEE EMBS San Francisco, USA, pp. 1620-1623, 2004.

[3]. Vallabha, D., Dorairaj, R., Namuduri, K., and Thompson, H., Automated detection and classification of vascular abnormalities in diabetic retinopathy, Proceedings of 13th IEEE Signals, Systems and Computers 2:1625-1629, 2004.

[4]. Sinthanayothin, C., Boyce, J., Williamson, T., Cook, H., Mensah, E., LaI, S., Usher, D., 2002. Automated detection of diabetic retinopathy on digital fundus images. Diabet. Med. 19,105- 112

[5]. Li, H., Chutatape, O., 2004. Automated feature extraction in color retinal images by a model based approach. IEEE Trans. Biomed. Eng. 5 1,246-254

[6]. Noronha, K., Nayak, J., and Bhat, S. Enhancement of retinal fundus image to highlight the features for detection of abnormal eyes. In Proceedings of the IEEE Region10 Conference (TENCON2006) (2006)

[7]. Lay, B., Baudoin, C., Klein, J.-C., 1983. Automatic detection of micro aneurysms in retinopathy fluoro-angiogram. Proc. SPIE 432,165.

[8]. Ege,B.,Hejlesen,Larsen,O.,Moller, K.,Jennings, B.,Kerr, D.,Cavan,D., 2000. Screening for diabetic retinopathy using computer based image analysis and statistical classification. Comput. Meth. Programs Biomed. 62,165- 175.

[9]. Lee, S., Lee, E., Kingsley, R., Wang, Y., Russell, D., Klein, R., Warn, A, 2001 Comparison of diagnosis of early retinal lesions of diabetic retinopathy between a computer and human experts. Arch. Ophthalmol.

[10]. Walter T, Massin P, Erginay A, Ordonez R, Jeulin C, Klein J-C "Automatic Detection of Micro aneurysms in Color Fundus Images Medical Image analysis"2007.

[11]. Niemeijer, M., van Ginneken, B., Russell, R. S., SuttorpSchulten,S. A. M., and Abramoff, D. M., Automated detection and differentiation of drusen, exudates, and cotton-wool spots in digital color fundus photographs for diabetic retinopathy diagnosis. Invest. Ophthalmol. Vis. Sci. 48(5):2260-2267, 2007.

[12]. Gardner, G., Keating, D., Williamson, T., Elliott, A., 1996. Automated detection of diabetic retinopathy using an 
artificial neural network: a screening tool. Br. J. Ophthalmol. 86,940-944

[13]. Bezdek,J.,Pal,M.,Keller,1.,Krisnapuram , R.,1999. Fuzzy Model and Algorithms for Pattern Recognition and Image Processing Kluwer Academic Press, London

[14]. Osareh, A., Mirmedhi, M., Thomas, B., Markham, R., 2003 Automated identification of diabetic retinal exudates in digital color imaging Br. J Ophthalmol 87, 1220- 1223

[15]. Acharya, U. R., Chua, K. C., Ng, E. Y. K., Wei, W., and Chee, C. Application of higher order spectra for the identification of diabetes retinopathy stages. J. Med. Systems, 2008, 32(6), 48 1-488

[16]. Kahai, P., Namuduri, K. R., and Thompson, H., A decision support framework for automated screening of diabetic retinopathy. Int. J. Biomed. Imag. 2006:1-8, 2006.

[17]. Wong, L. Y., Acharya, U. R., Venkatesh, Y. V., Chee, C., Lim, C. M., and Ng, E. Y. K., Identification of different stages of diabetic retinopathy using retinal optical images. Information Sciences 178(1):106-121, 2008.

[18]. Nayak, J., Bhat, P. S., Acharya, U. R., Lim, C. M., and Kagathi, M., Automated identification of different stages of diabetic retinopathy using digital fundus images. J. Med. Syst., USA, 32 (2):107-115, 2008.

[19]. Acharya, U. R., Lim, C. M., Ng, E. Y. K., Chee, C., and Tamura,T., Computer based detection of diabetes retinopathy stages using digital fundus images. J. Eng. Med. 223(H5):545553, 2009.

[20]. Adarsh. P and D. Jeyakumari, Multiclass SVM-Based Automated Diagnosis of Diabetic Retinopathy International conference on Communication and Signal Processing, April 35, 2013, India. 